\title{
Dirac composite fermion theory of general Jain sequences
}

\author{
Dung Xuan Nguyen $\oplus^{1}$ and Dam Thanh Son $\odot^{2}$ \\ ${ }^{1}$ Brown Theoretical Physics Center and Department of Physics, \\ Brown University, 182 Hope Street, Providence, Rhode Island 02912, USA \\ ${ }^{2}$ Kadanoff Center for Theoretical Physics, University of Chicago, Chicago, Illinois 60637, USA
}

(Received 9 August 2021; accepted 25 August 2021; published 7 September 2021)

\begin{abstract}
We reconsider the composite fermion theory of general Jain sequences with filling factor $v=N /(4 N \pm 1)$. We show that Goldman and Fradkin's proposal of a Dirac composite fermion leads to a violation of the Haldane bound on the coefficient of the static structure factor. To resolve this apparent contradiction, we add to the effective theory a gapped chiral mode (or modes) that already exists in the Fermi liquid state at $v=1 / 4$. We interpret the additional mode as an internal degree of freedom of the composite fermion, related to area-preserving deformations of the elementary droplet built up from electrons and correlation holes. In addition to providing a suitable static structure factor, our model also gives the expected Wen-Zee shift and a Hall conductivity that manifests Galilean invariance. We show that the charge density in the model satisfies the long-wavelength version of the Girvin-MacDonald-Platzman algebra.
\end{abstract}

DOI: 10.1103/PhysRevResearch.3.033217

\section{INTRODUCTION}

Since the discovery of the fractional quantum Hall effect (FQHE) in 1982 [1,2], many theoretical models have been invented to explain different aspects of this fascinating phenomenon. However, with no proposed theory capable of explaining all the richness of the $\mathrm{FQHE}$, it remains one of the most nontrivial questions of condensed-matter physics. One breakthrough idea was proposed by Jain [3], who suggested that the low-energy degree of freedom of the FQHE is the composite fermion (CF), which can be thought of as an electron moving together with an even number of magnetic flux quanta, a picture inspired by previous ideas [4-6]. The composite fermion model has achieved significant success. At the mean-field level, the composite fermion picture explains the presence (though not the magnitude) of the $\mathrm{FQH}$ gap, mapping it to an integer quantum Hall (IQH) gap of the composite fermions. Inspired by Jain's CF, Halperin, Lee, and Read introduced [7] a field theory-the HLR theory-that predicts a Fermi-liquid-like behavior of composite fermions near half-filling $\left(v=\frac{1}{2}\right)$, which was confirmed experimentally [8].

Despite its enormous success, the HLR theory leaves many issues unresolved. One issue is the scale of the energy gap, which is incorrectly predicted to be the cyclotron frequency in the simplest version of the flux attachment procedure. This issue can be resolved by treating the effective mass of the composite fermion as a phenomenological parameter, and by adding new terms in the effective Lagrangian, which, in the

Published by the American Physical Society under the terms of the Creative Commons Attribution 4.0 International license. Further distribution of this work must maintain attribution to the author(s) and the published article's title, journal citation, and DOI. lowest Landau level limit, amounts to assigning an electric dipole moment to the composite fermion. A more serious problem is the apparent breaking of particle-hole symmetry $(\mathrm{PH})$, which is the emergent symmetry of the lowest Landau level (LLL) [9]. Flux attachment equates the composite fermion density with the electron density, breaking the symmetry between electrons and holes, which holds in experiment [10]. The Dirac composite fermion theory [11] has been proposed as an effective field theory for $\mathrm{FQH}$ states near half-filling, which explicitly incorporates PH symmetry by assigning a Berry phase of $\pi$ for the CF around the Fermi surface. The theory gives reasonable predictions for the electromagnetic response functions [12].

In contrast to the case of $v=1 / 2$, at $v=1 / 4$ there is no symmetry that can be used to constrain the Berry phase of the CF. Recently, Goldman and Fradkin, motivated by the experimentally observed reflection symmetry relating the $I-V$ curves of states with filling fraction $v<1 / 4$ and $v>1 / 4$ $[8,10,13]$, proposed a Dirac composite fermion model for the $v=N /(4 N+1)$ Jain sequences (and for the general Jain sequences with $v=\frac{N}{2 n N \pm 1}$ ). The Berry phase of the $\mathrm{CF}$ in this model is equal to $\pi$. A similar effective theory for $\mathrm{FQH}$ states near $v=1 / 4$ was proposed by Wang in [14] motivated by the numerical study of the many-body Berry phase.

We will show in Goldman and Fradkin's model that the static structure factor of the $v=N /(4 N+1)$ [and, more generally, $v=N /(2 n N+1)]$ states violates the Haldane bound, which places a lower bound on the coefficient of the leading $q^{4}$ behavior of the projected static structure factor [15]

$$
s_{4} \geqslant \frac{|\mathcal{S}-1|}{8},
$$

where $s_{4}$ is the coefficient of $q^{4} \ell_{B}^{4}$ of the projected static structure factor and $\mathcal{S}$ is the Wen-Zee shift. This paper aims to construct an effective theory that adequately describes the physics of the $v=N /(4 N \pm 1)$ Jain sequences in the lowest 
Landau level limit. We combine the ideas of previous works [11,16-18], including Haldane's idea of a new geometric degree of freedom of the FQH system [17] and that of the bimetric theory of this degree of freedom [18]. Our model is a hybrid model that includes a Dirac composite fermion sector and an additional spin- 2 mode with the effective action of the form of the bimetric theory. In this model, in addition to the low-energy GMP mode at the energy scale of the effective cyclotron energy, which tends to zero as $N \rightarrow \infty$, there is an extra high-energy mode with energy that remains finite in the limit $N \rightarrow \infty$, and hence must already exist in the $v=1 / 2 n$ Fermi-liquid state. With the extra mode, which we call the Haldane mode, the issue with the Haldane bound of the projected static structure factor in the previous Dirac composite fermion model [16] can be fixed. We derive the topological Wen-Zee shift of Jain sequences that matches the expected results in the literature $[19,20]$. We also evaluate the wave-number dependence of the Hall conductivity and verify its connection with the Hall viscosity [21]. We then rederive the long-wavelength limit of GMP algebra as evidence that the theory is appropriate for the LLL limit.

The plan of the paper is as follows. In Sec. II we propose our effective theory, introducing its ingredients and briefly discussing the motivations. Section III is devoted to calculation of the Wen-Zee shift, the Hall viscosity, and the Hall conductivity. In Sec. IV, we derive the long-wavelength version of GMP algebra. In Sec. V we calculate the projected static structure factor using a semiclassical approach developed in Refs. [12,22], and we comment on the importance of the extra mode. Lastly, we conclude our paper and discuss the open questions in Sec. VI. Some technical details of the calculations are left to the Appendixes.

\section{EFFECTIVE FIELD THEORY}

Inspired by previous works [16-18,23], we propose an effective theory for the Jain sequences around $v=1 /(2 n)$, $\nu_{ \pm}=\frac{N}{2 n N \pm 1}$, where $n$ and $N$ are integers. In practice, Jain sequences have been seen only around $v=1 / 2$ and $1 / 4$, so in practice they can be seen for $n=2$, though for generality we will keep $n$ arbitrary in our formulas.

As we will see in Sec. V, the projected static structure factor for the $v=N /(4 N+1)$ calculated in the Dirac composite fermion model by Goldman and Fradkin [16] violates the Haldane bound. To fix this issue, we add an extra chiral mode to the model. Though the microscopic nature of this mode is not important for our effective theory, one can, following Haldane $[17,23]$, think about this mode as corresponding to the areapreserving deformation of the composite object ("elementary droplet") built up from electrons and correlation holes. In this paper, we show that with the proper choice of the coupling of the extra mode with external background electromagnetic field, we can reproduce the known physical results for the general Jain sequence.

\section{A. Symmetry of the lowest Landau level}

The system of electrons with Landé factor $\mathfrak{g}=2$ [24] in a constant magnetic field $B$ has a well-defined lowest Landau level (LLL) limit where the electron mass goes to zero [25,26].
Inheriting the symmetry of nonrelativistic massless fermions with $\mathfrak{g}=2[25,27,28]$, the external gauge field appears in the effective action as the combination

$$
\tilde{A}_{0}=A_{0}-\frac{1}{2} \epsilon^{i j} \partial_{i} v_{j}+\frac{1}{2} \omega_{0}, \quad \tilde{A}_{i}=A_{i}+\frac{1}{2} \omega_{i},
$$

where $v^{i}$ is the drift velocity, given by $v^{i}=\frac{\epsilon^{i j} E_{j}}{B}$ at the leading order, and $\omega_{\mu}$ is the spin connection of the Newton-Cartan space that was defined through derivative of vielbein $e_{i}^{a}$ [25],

$$
\begin{aligned}
& \omega_{0}=\frac{1}{2} \epsilon^{i j} \partial_{i} v_{j}+\frac{1}{2} \epsilon^{a b} e^{a j} \partial_{0} e_{j}^{b}, \\
& \omega_{i}=\frac{1}{2} \epsilon^{a b} e^{a j} \partial_{i} e_{j}^{b}-\frac{1}{2} \epsilon^{j k} \partial_{j} g_{i k} .
\end{aligned}
$$

In particular, in flat space and uniform magnetic field,

$$
\tilde{A}_{0}=\left(1+\frac{\nabla^{2}}{4 B}+\cdots\right) A_{0} .
$$

Effective theories on the LLL, including composite fermion models, should couple with the external gauge field through $\tilde{A}_{\mu}$.

\section{B. Composite fermion sector}

In this paper, we propose the following effective action of Jain sequences:

$$
S=S_{\mathrm{CF}}+S_{\text {Haldane }},
$$

where the Dirac composite fermion action is given by

$$
\begin{aligned}
S_{\mathrm{CF}}= & \int d^{3} x \sqrt{g} \frac{i}{2}\left(\psi^{\dagger} \overleftrightarrow{D_{t}} \psi+e_{a}^{i} \psi^{\dagger} \sigma^{a} \overleftrightarrow{D}_{i} \psi+v^{i} \psi^{\dagger} \overleftrightarrow{D_{i}} \psi\right) \\
& \times \int\left[-\frac{1}{8 \pi}\left(1-\frac{1}{n}\right) a d a-\frac{1}{4 \pi n} \tilde{A} d a+\frac{1}{8 \pi n} \tilde{A} d \tilde{A}\right]
\end{aligned}
$$

where $\sigma^{a}$ is the Pauli matrix and $a_{\mu}$ is the emergent gauge field. As in Ref. [16], the Dirac composite fermion is massless. Here we chose the Fermi velocity of composite fermion $v_{F}=1$ to simplify the formulas [12]; the main results of this paper do not depend on $v_{F}$. The covariant derivative of the composite fermion is

$$
D_{\mu} \psi \equiv\left(\partial_{\mu}-i a_{\mu}+\frac{i}{2} \sigma^{3} \omega_{\mu}\right) \psi .
$$

The CF is charge-neutral without direct coupling to the external electromagnetic field. The third term of (7) implies that the composite fermion has a dipole moment perpendicular to its momentum. This term is new compared to previous work on the Dirac composite fermion model with the filling fraction near $1 / 2 n[14,16]$. The neutrality of the composite fermions, as well as its dipole moment, are features of the Dirac composite fermion near half-filling [11,12].

Differentiating the action over $a_{0}$ gives us a constraint (we work in flat space)

$$
\bar{\rho}_{\mathrm{CF}}=\frac{1}{4 \pi}\left(1-\frac{1}{n}\right) \bar{b}+\frac{1}{4 \pi n} B,
$$

while differentiating the action with respect to $A_{0}$ and taking the average over space gives the average physical charge 
density,

$$
\bar{\rho}_{e}=\frac{1}{4 \pi n} B-\frac{1}{4 \pi n} \bar{b} .
$$

The Haldane mode will not change the above equation; physically, the area-preserving deformations of the elementary droplets do not change the average density. The specific values of emergent magnetic field

$$
\bar{b}= \pm \frac{1}{2 n N \pm 1} B
$$

correspond to $\mathrm{FQH}$ filling fractions of the Jain states,

$$
v_{ \pm}=\frac{N}{2 n N \pm 1},
$$

where the filling fraction of the composite fermion is

$$
\nu_{ \pm}^{\mathrm{CF}}= \pm N+\frac{1}{2},
$$

which are the filling factors of IQH states of a massless Dirac fermion.

\section{Haldane sector}

The Haldane mode (so named because it embodies Haldane's idea of an emergent degree of freedom of the FQHE $[17,23])$ is a gapped dynamical spin-2 degree of freedom described by the extra metric $\hat{g}_{i j}$. We define the emergent vielbein as the square root of the ambient metric,

$$
\hat{g}_{i j}=\hat{e}_{i}^{\alpha} \hat{e}_{j}^{\beta} \delta_{\alpha \beta},
$$

and we also denote the matrix inverse of $\hat{e}_{i}^{\alpha}$ by $\hat{E}_{\alpha}^{i}$. The compatibility conditions,

$$
\begin{aligned}
& \hat{\nabla}_{k} \hat{g}_{i j}=\partial_{k} \hat{g}_{i j}-\hat{\Gamma}_{k, i}^{i} \hat{g}_{i j}-\hat{\Gamma}_{k, j}^{l} \hat{g}_{l j}=0, \\
& \hat{\nabla}_{\mu} \hat{e}_{v}^{A}=\partial_{\mu} \hat{e}_{v}^{A}-\hat{\Gamma}_{v, \mu}^{\lambda} \hat{e}_{\lambda}^{A}+\hat{\omega}_{B, \mu}^{A} \hat{e}_{v}^{B}=0,
\end{aligned}
$$

are satisfied by

$$
\begin{gathered}
\hat{\omega}_{0}=\frac{1}{2} \epsilon^{\alpha \beta} \hat{E}_{\beta}^{i} \partial_{0} \hat{e}_{i}^{\alpha}, \\
\hat{\omega}_{j}=\frac{1}{2} \epsilon^{\alpha \beta}\left(\hat{E}_{\beta}^{i} \partial_{j} \hat{e}_{i}^{\alpha}-\hat{\Gamma}_{i, j}^{k} \hat{e}_{k}^{\alpha} \hat{E}_{\beta}^{i}\right), \\
\hat{\Gamma}_{k, j}^{i}=\frac{1}{2} \hat{G}^{i l}\left(\partial_{j} \hat{g}_{k l}+\partial_{k} \hat{g}_{j l}-\partial_{l} \hat{g}_{j k}\right), \\
\hat{\Gamma}_{j, 0}^{i}=\frac{1}{2} \hat{G}^{i k} \partial_{0} \hat{g}_{j k} .
\end{gathered}
$$

We further introduce the definition of the emergent Ricci curvature of $\hat{g}_{i j}$ as

$$
\hat{R}=\frac{2}{\sqrt{\hat{g}}}\left(\partial_{1} \hat{\omega}_{2}-\partial_{2} \hat{\omega}_{1}\right) .
$$

Restricting the dynamical part of the the emergent metric to fluctuations that preserve the area, setting $\hat{g}=g$, we can rewrite the emergent Ricci curvature as

$$
\hat{R}=\frac{2}{\sqrt{g}}\left(\partial_{1} \hat{\omega}_{2}-\partial_{2} \hat{\omega}_{1}\right) .
$$

We propose to use as the action of the spin- 2 mode the leading (in derivatives) terms of bimetric theory introduced in
Refs. [18,29],

$$
\begin{aligned}
S_{\text {Haldane }}= & \int \frac{\zeta}{4 n \pi} A d \hat{\omega}-\int d^{3} x \sqrt{g}\left[\frac{\tilde{m}}{2}\left(\frac{1}{2} \hat{g}_{i j} g^{i j}-\gamma\right)^{2}\right. \\
& \left.+\frac{\zeta}{8 n \pi B} g^{i j}\left(\partial_{i} E_{j}\right) \mathcal{B}\right],
\end{aligned}
$$

with $v$ is the FQH filling fraction, and the magnetic field in curved space is defined as $\mathcal{B}=\frac{\partial_{1} A_{2}-\partial_{2} A_{1}}{\sqrt{g}}$. The mass $\tilde{m}>$ 0 in the potential term sets the energy gap for the extra spin-2 mode. We require $\gamma<1$ so that the ground state is isotropic [18,29]. The last term comes from the symmetry of LLL, where the temporal component of the emergent spin connection enters the Lagrangian through the combination $\hat{\omega}_{0}+\frac{1}{2} \epsilon^{i j} \partial_{i} v_{j}$, hence its coefficient is fixed by that of the first term. This last term contributes to the finite-wave-number Hall conductivity at the $k^{2}$ order. Besides $\tilde{m}$ there is only one additional parameter, $\zeta$ [18]. In the subsequent section, we will show that one can fix the coefficient $\zeta$ for general Jain sequences using the Wen-Zee shift. We will find that $\zeta$ only depends on $n$ but not $N$, as expected.

\section{WEN-ZEE SHIFT $\mathcal{S}$ AND GALILEAN INVARIANT}

In this section, we find the value of $\zeta$. We show that, with $\zeta=n-1$, one can obtain the expected Wen-Zee shift, the finite-wave-number Hall conductivity, and the Hall viscosity for the general Jain sequences $v_{ \pm}$. The results demonstrate that the topological Wen-Zee shift as well as the relationship between the finite-wave-number Hall conductivity and the shift [21] are reproduced successfully in our model.

\section{A. Wen-Zee shift $\mathcal{S}$}

\section{Filling fraction $v_{+}=\frac{N}{2 n N+1}$}

At the filling fraction $v_{+}$, the filling fraction of the composite fermion is $v^{\mathrm{CF}}=N+1 / 2$. The Dirac composite fermion then forms an IQH state. One can then integrate out the fermion fields $\psi$ and obtain the Chern-Simons (CS) action

$$
\frac{N+1 / 2}{4 \pi} a d a+\frac{N(N+1)}{4 \pi} a d \omega .
$$

The first term encodes the Hall conductivity of the IQH state of the Dirac fermion [30]. The second term represents the effective coupling of Dirac composite fermions with the background curvature, which reproduces the known Wen-Zee shift of Dirac IQH states [27,31,32]. We have the following leading terms in the effective Lagrangian of the composite fermion sector [33]:

$$
\begin{aligned}
\mathcal{L}_{\mathrm{CF}}= & \frac{N+1 / 2}{4 \pi} a d a+\frac{N(N+1)}{4 \pi} a d \omega-\frac{1}{8 \pi}\left(1-\frac{1}{n}\right) a d a \\
& -\frac{1}{4 \pi n} \tilde{A} d a+\frac{1}{8 \pi n} \tilde{A} d \tilde{A} .
\end{aligned}
$$

We then integrate out the emergent gauge field $a_{\mu}$ to obtain the final effective action of external background fields,

$$
\mathcal{L}_{\mathrm{CF}}=-\frac{n N^{2}(N+1)^{2}}{8 \pi(2 n N+1)} \omega d \omega+\frac{\nu_{+}}{4 \pi}(N+1) \tilde{A} d \omega \dagger \frac{\nu_{+}}{4 \pi} \tilde{A} d \tilde{A} .
$$


From Eq. (26) we obtain the contribution to the charge density from the Dirac composite sector in a static background without electric field [34],

$$
\bar{\rho}^{(1)}=\frac{\nu_{+}}{2 \pi} \mathcal{B}+\frac{\nu_{+}(N+2)}{8 \pi} R,
$$

with $R \equiv \frac{2}{\sqrt{g}}\left(\partial_{1} \omega_{2}-\partial_{2} \omega_{1}\right)$ being the Ricci curvature of the background metric $g_{i j}$. The first term of (27) comes from the electromagnetic CS term $\tilde{A} d \tilde{A}$. The second term receives contributions from the mixed CS term $\tilde{A} d \omega$ and the $\tilde{A} d \tilde{A}$ tern due to the spin connection in the definition of $\tilde{A}$ [35].

Now let us consider the response of the Haldane sector to background fields. At zero frequency, in the isotropic phase, the potential term in (23) constrains the ambient metric to follow the background metric $\hat{g}_{i j}=g_{i j}$ [18]. As a consequence, one can read off the contribution to charge density of the Haldane sector in a static background without an electric field,

$$
\bar{\rho}^{(2)}=\frac{\zeta}{8 n \pi} \hat{R}=\frac{\zeta}{8 n \pi} R .
$$

Combining Eqs. (27) and (28), we obtain the charge density,

$$
\rho_{e}=\frac{\nu_{+}}{2 \pi} \mathcal{B}+\frac{\nu_{+}(N+2)}{8 \pi} R+\frac{\zeta}{8 n \pi} R,
$$

and from it the total charge on a closed manifold,

$$
N_{e}=v_{+}\left[N_{\phi}+(N+2+2 \zeta) \frac{\chi}{2}\right]+O\left(\frac{1}{N}\right),
$$

where $N_{\phi}$ is the total number of flux quanta $N_{\phi}=\frac{1}{2 \pi} \int \sqrt{g} \mathcal{B}$, and $\chi$ is the Euler character of the Riemann surface,

$$
\chi=\frac{1}{4 \pi} \int \sqrt{g} R,
$$

which means that the Wen-Zee shift [36] is

$$
\mathcal{S}_{\nu_{+}}=N+2+2 \zeta,
$$

where we dropped the term of subsub leading in the $1 / N$ expansion.

\section{Filling fraction $v_{-}=\frac{N}{2 n N-1}$}

One can repeat the calculations for $\nu_{+}$and obtain the effective Lagrangian of the composite fermion sector for $v_{-}$,

$\mathcal{L}_{\mathrm{CF}}=-\frac{n N^{2}(N-1)^{2}}{8 \pi(2 n N-1)} \omega d \omega+\frac{\nu_{-}}{4 \pi}(-N+1) \tilde{A} d \omega \dagger \frac{\nu_{-}}{4 \pi} \tilde{A} d \tilde{A}$.

We again combine the charge density of the CF sector and the Haldane sector and obtain

$$
\rho_{e}=\frac{\nu_{-}}{2 \pi} \mathcal{B}+\frac{\nu_{-}(-N+2)}{8 \pi} R+\frac{\zeta}{8 n \pi} R .
$$

The first two terms of (34) come from the effective Lagrangian of composite fermion sector (33), while the last term comes from the Haldane sector (23). We then obtain the Wen-Zee shift up to leading and first subleading orders in the $1 / N$ expansion,

$$
\mathcal{S}_{\nu_{-}}=-N+2+2 \zeta .
$$

We now see that if we choose

$$
\zeta=n-1
$$

we can reproduce the expected Wen-Zee shift for both $v_{+}$and $v_{-}$sequences $[19,20]$,

$$
\mathcal{S}_{v_{+}}=N+2 n, \quad \mathcal{S}_{\nu_{-}}=-N+2 n .
$$

For Jain sequences near half-filling with $n=1$, the coefficient $\zeta=0$. In this case, there is no need for a Haldane mode. We argue that, due to the particle-hole symmetry at $v=1 / 2$, the electrons and holes are uniformly distributed in the elementary droplets, so their area-preserving deformations do not change the local charge density and then decouple from the external electromagnetic field.

\section{B. Hall viscosity}

To derive the zero-frequency Hall viscosity, one needs to work out the coefficient of the mixed CS term $A d \omega$ in the effective action. This coefficient is determined by the WenZee shift as $v \mathcal{S} A d \omega$ [36]. One can also do the calculation explicitly using the effective Lagrangian of the CF sector and replace, in the Haldane sector, the emergent spin connection $\hat{\omega}$ by the spin connection of the background metric $\omega$ at low energy. We found that

$$
\eta_{0}=\bar{\rho}_{e} \frac{\mathcal{S}}{4}
$$

for both sequences $v_{-}$and $v_{+}$.

\section{Finite-wave-number Hall conductivity}

In a flat background where $g_{i j}=\delta_{i j}$, using the definitions of $\omega_{0}$ and $\tilde{A}$, we can rewrite the CF action (26) as

$$
\mathcal{L}_{\mathrm{CF}}=-\frac{\nu_{+}}{8 \pi B} N(\vec{\nabla} \times \vec{A}) \partial_{i} E_{i}+\frac{\nu_{+}}{4 \pi} A d A .
$$

The Lagrangian of the Haldane sector for Jain's sequence $v_{+}$ state at low energy can be rewritten as

$$
\mathcal{L}_{\text {Haldane }}=-\frac{\zeta}{8 n \pi B}(\vec{\nabla} \times \vec{A}) \partial_{i} E_{i} .
$$

One can derive the current in flat space, with $\zeta=n-1$, as a function of the applied electric field (we work up to leading and first subleading order in the $1 / N$ expansion),

$$
J^{i}=\frac{\nu_{+}}{2 \pi} \epsilon^{i j} E_{j}-\frac{\nu_{+}}{4 \pi B}\left(\mathcal{S}_{\nu_{+}}-\mathfrak{g}\right) \epsilon^{i j} \partial_{j}(\vec{\nabla} \cdot \vec{E}),
$$

where we have assumed the Landé factor of the electron is $\mathfrak{g}=$ 2. We then obtain the Hall conductivity at finite momentum,

$$
\sigma_{H}^{\nu_{+}}(q)=\frac{\nu_{+}}{2 \pi}\left(1+\frac{\mathcal{S}_{\nu_{+}}-\mathfrak{g}}{4} q^{2} \ell_{B}^{2}\right),
$$

where the magnetic length is $\ell_{B}=1 / \sqrt{B}$. One can perform the same calculation to yield the finite momentum Hall conductivity of Jain's sequence $v_{-}$,

$$
\sigma_{H}^{\nu_{-}}(q)=\frac{\nu_{-}}{2 \pi}\left(1+\frac{\mathcal{S}_{\nu_{-}}-\mathfrak{g}}{4} q^{2} \ell_{B}^{2}\right) .
$$

The wave-number dependence of the Hall conductivity of both sequences $v_{+}$and $v_{-}$satisfies the relationship with the Wen-Zee shift [21]. The results imply that our model satisfies the symmetries of the LLL, as designed. 


\section{THE GMP ALGEBRA}

In the classic paper [37], Girvin, MacDonald, and Platzman showed that the LLL projected density operator $\rho_{e}(\mathbf{k})$ obeys the following (GMP) algebra:

$$
[\rho(\mathbf{k}), \rho(\mathbf{q})]=2 i e^{\frac{1}{2}(\mathbf{k} \cdot \mathbf{q}) \ell_{B}^{2}} \sin \left(\frac{\mathbf{k} \times \mathbf{q}}{2} \ell_{B}^{2}\right) \rho(\mathbf{k}+\mathbf{q}) .
$$

The GMP algebra was discovered independently in string theory with the name $W_{\infty}[38,39]$. In the FQHE, the GMP algebra is a consequence of charge conservation and the LLL constraint. In the long-wavelength approximation, the GMP algebra is reduced to the algebra of area-preserving diffeomorphisms [40],

$$
[\rho(\mathbf{k}), \rho(\mathbf{q})] \approx i(\mathbf{k} \times \mathbf{q}) \ell_{B}^{2} \rho(\mathbf{k}+\mathbf{q}) .
$$

Near half-filling, the long-wavelength limit of GMP algebra has been shown to arise from Dirac composite fermion theory in Ref. [12]. In this section, we generalize the derivation of the GMP algebra for the general Jain sequences. From the action (6), one can read off the charge density operator in the flat background where $g_{i j}=\delta_{i j}, \hat{R}=2 \epsilon^{i j} \partial_{i} \hat{\omega}_{j}$ up to subleading order in the momentum expansion,

$$
\rho_{e}=\rho^{(1)}+\rho^{(2)}
$$

where $\rho^{(1)}$ and $\rho^{(2)}$ come from the CF and the Haldane sectors, respectively. Explicitly,

$$
\begin{gathered}
\rho^{(1)}=\frac{B-b}{4 \pi n}-\frac{\epsilon^{i j}}{B} \partial_{i} \Pi_{j}, \\
\rho^{(2)}=\frac{\zeta}{8 n \pi} \hat{R} .
\end{gathered}
$$

The second term of Eq. (47) implies that the composite fermion has a dipole moment perpendicular and proportional to its momentum. Employing the canonical equal-time anticommutation relation of the CF field,

$$
\left\{\psi(\mathbf{x}), \psi^{\dagger}(\mathbf{y})\right\}=\delta(\mathbf{x}-\mathbf{y}),
$$

and the commutation relation of the emergent gauge field due to the Chern-Simon term of $a_{\mu}$ in the action (7),

$$
\left[a_{i}(\mathbf{x}), a_{j}(\mathbf{y})\right]=-i \frac{4 \pi n}{n-1} \epsilon^{i j} \delta(\mathbf{x}-\mathbf{y}),
$$

one can derive the algebra up to lowest order in momentum of $\rho^{(1)}$,

$$
\left[\rho^{(1)}(\mathbf{k}), \rho^{(1)}(\mathbf{q})\right]=i(\mathbf{k} \times \mathbf{q}) \ell_{B}^{2} \rho^{(1)}(\mathbf{k}+\mathbf{q}) .
$$

The interested reader can find the detailed derivation of Eq. (51) in Appendix B. Equation (51) implies the GMP algebra of the CF sector.

Following Ref. [18], one finds that the charge density in the Haldane sector also satisfies the GMP algebra,

$$
\left[\rho^{(2)}(\mathbf{k}), \rho^{(2)}(\mathbf{q})\right]=i(\mathbf{k} \times \mathbf{q}) \ell_{B}^{2} \rho^{(2)}(\mathbf{k}+\mathbf{q}) .
$$

Combining Eqs. (51) and (52), we arrive at the GMP algebra of the electron density operator at the lowest order in momentum,

$$
\left[\rho_{e}(\mathbf{k}), \rho_{e}(\mathbf{q})\right]=i(\mathbf{k} \times \mathbf{q}) \ell_{B}^{2} \rho_{e}(\mathbf{k}+\mathbf{q}) .
$$

The GMP algebra is the key feature of a LLL effective theory. For the algebra, it is crucial for the Dirac composite fermion to have an electric dipole moment [12]. The dipole moment term in Eq. (7) thus not only preserves the LLL invariance of the DCF action but also plays an essential role in the GMP algebra (53).

\section{DYNAMICS OF THE CF SURFACE AND THE STATIC STRUCTURE FACTOR}

In the theory of a Dirac composite fermion near half-filling, the dynamics of the composite fermion sector at low energy can be interpreted as the deformation of the composite Fermi surface $[12,22,29,41]$. One can apply the semiclassical equation of motion to analyze the physics at the long-wavelength limit [42]. In this section, we will extend the semiclassical approach for Dirac composite fermion theory of the general Jain sequences and derive the static structure factor (SSF).

\section{A. Semiclassical equation of motions}

Near the filling fraction $v=\frac{1}{2 n}$, the Dirac composite fermion forms the Fermi surface with a small effective background magnetic field $\bar{b}$ induced by the emergent gauge $a_{\mu}$. Following Refs. [12,22,29,41], we introduce the deformation of the composite Fermi surface at a given position $\mathbf{x}$,

$$
k_{F}(\theta, \mathbf{x})=k_{F}+\sum_{m=-\infty}^{\infty} u_{m}(\mathbf{x}) e^{-i m \theta},
$$

where $u_{n}(\mathbf{x})$ is a space-dependent function and $\theta$ is the polar angle in momentum space. The Fermi momentum is related to the composite fermion density by Luttinger's theorem [43],

$$
\frac{k_{F}^{2}}{4 \pi}=\bar{\rho}_{\mathrm{CF}}=\frac{1}{4 \pi}\left(1-\frac{1}{n}\right) \bar{b}+\frac{1}{4 \pi n} B .
$$

$u_{1}(\mathbf{x})$ and $u_{-1}(\mathbf{x})$ can be regarded as the complex components of a vector $u_{i}(\mathbf{x})$,

$$
u_{1}=u_{\bar{z}}, \quad u_{-1}=u_{z},
$$

where we defined the complex coordinate

$$
z=x+i y, \quad \bar{z}=x-i y,
$$

and the complex derivative

$$
\partial_{z}=\frac{1}{2}\left(\partial_{x}-i \partial_{y}\right), \quad \partial_{\bar{z}}=\frac{1}{2}\left(\partial_{x}+i \partial_{y}\right) .
$$

The composite fermion density and momentum operator in this essentially semiclassical formalism are [12,22]

$$
\rho_{\mathrm{CF}}=\bar{\rho}_{\mathrm{CF}}+\frac{k_{F}}{2 \pi} u_{0}, \quad \Pi_{i}=\frac{k_{F}^{2}}{4 \pi} u_{i} .
$$

The $u_{n}$ mode satisfies the following commutation relation $[22,41]$ :

$$
\begin{aligned}
& {\left[u_{m}(\mathbf{x}), u_{m^{\prime}}\left(\mathbf{x}^{\prime}\right)\right]} \\
& \quad=\frac{2 \pi}{k_{F}}\left(\frac{m \bar{b}}{k_{F}} \delta_{m+m^{\prime}, 0}-i \delta_{m+m^{\prime}, 1} \partial_{\bar{z}}-i \delta_{m+m^{\prime},-1} \partial_{z}\right) \delta\left(\mathbf{x}-\mathbf{x}^{\prime}\right) .
\end{aligned}
$$

The same commutation relation for the Fermi surface in a magnetic field was derived by Haldane using bosonization 
[44]. The Fermi-liquid kinetic equation can be interpreted as the equations of motion that followed from the commutation relation (60) and the Hamiltonian [12,45]

$$
H=\frac{v_{F} k_{F}}{4 \pi} \sum_{m=-\infty}^{\infty} \int d \mathbf{x}\left(1+F_{m}\right) u_{m}(\mathbf{x}) u_{-m}(\mathbf{x})-a_{0} \rho_{\mathrm{CF}}
$$

where $F_{m}$ are the Landau Fermi-liquid parameters. The Heisenberg equation of motion of $u_{m}(\mathbf{x})$ is [46]

$$
\begin{aligned}
\dot{u}_{m}= & -i\left(1+F_{m}\right) \frac{\bar{b} v_{F}}{k_{F}} u_{m}-v_{F}\left(1+F_{m-1}\right) \partial_{\bar{z}} u_{m-1} \\
& -v_{F}\left(1+F_{m+1}\right) u_{m+1}+\delta_{m, 1} e_{\bar{z}}+\delta_{m,-1} e_{z},
\end{aligned}
$$

with $e_{i}=\partial_{i} a_{0}-\partial_{0} a_{i}$. For $m=0$, Eq. (62) gives

$$
\dot{u}_{0}=-v_{F}\left(1+F_{1}\right)\left(\partial_{\bar{z}} u_{-1}+\partial_{z} u_{1}\right),
$$

which can be understood as the conservation law of the $\mathrm{CF}$ number: $\partial_{t} \rho_{\mathrm{CF}}+\nabla \cdot \mathbf{j}_{\mathrm{CF}}=\mathbf{0}$, with CF number current

$$
j_{i}^{\mathrm{CF}}=\frac{v_{F}\left(1+F_{1}\right)}{k_{F}} \Pi_{i} .
$$

We will use this semiclassical approach to derive the density-density correlation function, and specifically the static structure factor to leading order in the momentum expansion.

\section{B. Projected static structure factor}

In this section, we will use the semiclassical approach to calculate the leading order in gradient expansion of the projected static structure factor. We will show that the extra Haldane mode is crucial to satisfy the Haldane bound, and the fact that the latter is satisfied provides a nontrivial check for our proposal.

We combine Eqs. (47) and (59) to obtain the charge density operator of the $\mathrm{CF}$ sector in the semiclassical formalism,

$$
\rho^{(1)}=\frac{B-b}{4 \pi n}+\frac{i k_{F}^{2}}{2 \pi B}\left(\partial_{z} u_{1}-\partial_{\bar{z}} u_{-1}\right) .
$$

We take the time derivative of the above equation and use the equation of motion (62) for $m=0, \pm 1, \pm 2$ to obtain, up to leading order in the gradient expansion,

$$
\dot{\rho}^{(1)}=\frac{\dot{b}}{4 \pi}\left(\frac{k_{F}^{2}}{B}-\frac{1}{n}-\frac{\bar{b}}{B} \frac{n-1}{n}\right)+\frac{k_{F}^{3}}{4 \pi B \bar{b}}\left(\partial_{z}^{2} \dot{u}_{2}+\partial_{\bar{z}}^{2} \dot{u}_{-2}\right) .
$$

We leave the detail derivation of the above equation to Appendix C. Using the explicit value of $k_{F}$ from Eq. (55), we see that only the term including $u_{ \pm 2}$ in (66) survives, and we are left with

$$
\delta \rho^{(1)}=\frac{k_{F}^{3}}{4 \pi B \bar{b}}\left(\partial_{z}^{2} u_{2}+\partial_{\bar{z}}^{2} u_{-2}\right) .
$$

Note that by the construction of the Hamiltonian (61), at the long-wavelength limit the ground state is annihilated by $u_{m}$ with $m<0$ (see Ref. [22] for details): $u_{-2}|0\rangle=\langle 0| u_{2}=0$, which allows us to derive the equal-time density-density correlation function,

$$
\left\langle\delta \rho^{(1)}(-\mathbf{q}) \delta \rho^{(1)}(\mathbf{q})\right\rangle=q^{4} \frac{k_{F}^{6}}{256 \pi^{2} B^{2} \bar{b}^{2}}\left\langle\left[u_{-2}(-\mathbf{q}), u_{2}(\mathbf{q})\right]\right\rangle .
$$

We then use the commutation relation of $u_{ \pm 2}$ and obtain

$$
\left\langle\delta \rho^{(1)}(-\mathbf{q}) \delta \rho^{(1)}(\mathbf{q})\right\rangle=\frac{k_{F}^{4}}{64 \pi B^{2}|\bar{b}|} q^{4}=\frac{\pi}{4} \frac{\bar{\rho}_{\mathrm{CF}}^{2}}{|\bar{b}|}\left(q \ell_{B}\right)^{4} .
$$

From Eq. (69) we then read off the contribution to the coefficient of $q^{4} \ell_{B}^{4}$ of the projected SSF from the CF sector,

$$
\bar{s}_{4}^{(1)}=\frac{1}{8} \frac{\bar{\rho}_{\mathrm{CF}}^{2}}{\bar{\rho}_{e}} \frac{2 \pi}{|\bar{b}|},
$$

where we defined the projected SSF following Girvin, MacDonald, and Platzman [37],

$$
\bar{s}=\frac{1}{\bar{\rho}_{e}}\langle\delta \rho(-\mathbf{q}) \delta \rho(\mathbf{q})\rangle .
$$

From the action of the Haldane sector (23), one can calculate the contribution to the equal time correlation function $\left\langle\delta \rho^{(2)}(-\mathbf{q}) \delta \rho^{(2)}(\mathbf{q})\right\rangle$ as well as the contribution of the Haldane sector to the projected SSF. The calculation follows directly from Ref. [18], we will not repeat the calculation and quote the result only:

$$
\bar{s}_{4}^{(2)}=\frac{2 \zeta}{16 n v_{+}} .
$$

We then have the total static structure factor [47]

$$
\bar{s}_{4}=\bar{s}_{4}^{(1)}+\bar{s}_{4}^{(2)}=\frac{1}{8}\left(\frac{\bar{\rho}_{\mathrm{CF}}^{2}}{\bar{\rho}_{e}} \frac{2 \pi}{|\bar{b}|}+\frac{2 \zeta}{2 n \nu_{+}}\right) .
$$

We are now in a position to compute the coefficient $\bar{s}_{4}$ for both filling fractions $\nu_{ \pm}$of the general Jain sequences. Combining Eqs. (73), (9), (10), and (11), one obtains, for filling fraction $v_{+}=\frac{N}{2 n N+1}$,

$$
\bar{s}_{4}^{\nu_{+}}=\frac{1}{8}(N+1+2 \zeta)+O\left(\frac{1}{N}\right),
$$

and for $v_{-}=\frac{N}{2 n N-1}$

$$
\bar{s}_{4}^{\nu_{-}}=\frac{1}{8}(N-1+2 \zeta)+O\left(\frac{1}{N}\right) .
$$

The $O(1 / N)$ corrections in Eqs. (74) and (75) are beyond the limit of accuracy of the semiclassical approximation and will be dropped in future formulas. If we fix the coefficient $\zeta=$ $n-1$ as in Sec. III to match the Wen-Zee shift, we finally have the coefficient $\bar{s}_{4}$,

$$
\begin{aligned}
& \bar{s}_{4}^{\nu_{+}}=\frac{1}{8}(N+2 n-1), \\
& \bar{s}_{4}^{\nu_{-}}=\frac{1}{8}(N-3+2 n) .
\end{aligned}
$$

Remembering that the shift of $v_{+}$is $\mathcal{S}_{v_{+}}=N+2 n$, we see that $\bar{s}_{4}^{\nu_{+}}$saturates the Haldane bound

$$
\bar{s}_{4}^{\nu_{+}}=\frac{\mathcal{S}_{v_{+}}-1}{8},
$$

which suggests that $v_{+}$is a chiral state $[17,48]$. However, the Haldane bound for the $v_{-}$state,

$$
\bar{s}_{4}^{\nu_{-}} \geqslant \frac{\left|\mathcal{S}_{\nu_{-}}-1\right|}{8},
$$

is saturated only for $n=1$ [49]. For example, the $v=$ $N /(4 N-1)$ states are not chiral: the chirality of the Haldane 
mode is opposite to the chirality of the low-energy magnetoroton.

We emphasize here that the contribution to the projected SSF of the Haldane sector is crucial in our proposal. Without the additional term $\bar{s}_{4}^{(2)}$, the projected SSF of the Jain sequence $v_{+}$violates the Haldane bound for $n>1$ [17,23]. The coefficient $\zeta=n-1$ not only helps us to obtain the correct Wen-Zee shift in Sec. III, but it also makes it possible to satisfy the lower bound on the projected SSF.

\section{CONCLUSION}

In this paper, we propose a Dirac composite fermion model of general Jain sequences $v=\frac{N}{2 n N \pm 1}$. The proposal incorporates a Dirac composite fermion and an extra spin- 2 mode at $v=1 / 4$ and the Jain sequences around it. We show that our model reproduces the known results of Hall conductivity and Hall viscosity. The results imply that our model satisfies the Galilean invariant and reproduces the Wen-Zee shift. Using the semiclassical approach, we calculated the coefficient $\bar{s}_{4}$ of the projected static structure factor. We show that, without the extra mode, the projected SSF violates the Haldane bound. We also derive the long-wavelength limit of the GMP algebra that emerges from the model. In summary, the results calculated in this paper suggest that our Dirac composite fermion model is a proper effective theory of LLL that provides the expected physical results of general Jain sequences.

In this paper, we proposed a single Haldane mode with spin-2. However, we can come up with the Haldane sector that includes multiple extra spin-2 modes. In particular, if we consider $s$ spin-2 modes with different energy gaps determined by $\tilde{m}_{i}$ and different coupling $\zeta_{i}$ with background electromagnetic field as described in Eq. (23), then we can reproduce the physical results of this paper with the condition

$$
\sum_{i=-1}^{s} \zeta_{i}=n-1
$$

We then arrive at the other main conclusion of this paper, which is the extra spin-2 mode(s). We expect that those modes have higher energy gaps than the Dirac composite fermion effective cyclotron frequency but still lower than the electron's cyclotron gap. We suggest that the appearance of the extra spin-2 mode(s) can be confirmed in the numerical calculation of the correlation function of the stress tensor operator in the LLL $[50,51]$ at or near the filling fraction $\frac{1}{4}$. We expect that there would be an additional peak(s) in the spectral function of the stress tensor at high frequency. We can also verify the extra spin-2 mode(s) with their chiralities by circularly polarized Raman scattering [52].

In this paper, we assume that the Berry phase of the composite fermion is equal to $\pi$ as for a massless Dirac fermion, a choice motivated by the symmetry of the $I-V$ curve around $v=1 / 4$. Without this motivation, one can consider other possibilities; for example, one in which the CF has zero Berry phase as in the HLR theory. The Haldane mode will also be needed here to avoid violation of the Haldane bound within the HLR theory (which already occurs at near half-filling [12]).

\section{ACKNOWLEDGMENTS}

We thank Andrey Gromov, Duncan Haldane, Ed Rezayi, and Kun Yang for discussions. D.T.S. is supported in part by U.S. DOE Grant No. DE-FG02-13ER41958, a Simons Investigator grant, and by the Simons Collaboration on Ultra-Quantum Matter, which is a grant from the Simons Foundation (651440, DTS). D.X.N. was supported by Brown Theoretical Physics Center.

\section{APPENDIX A: SPECTRAL SUM RULES AND THE HALDANE BOUND}

For completeness, we present the derivation of the Haldane bound in this Appendix. 2D electrons in a constant magnetic field satisfies the following Ward's identities:

$$
\begin{gathered}
\partial_{t} \rho+\nabla \cdot \mathbf{j}=\mathbf{0}, \\
\partial_{t}\left(m j_{i}\right)+\partial_{k} T_{k i}=(\mathbf{j} \times \mathbf{B})_{i},
\end{gathered}
$$

which are merely the conservations of $U(1)$ charge and momentum density, where the momentum density of the nonrelativistic electron is $P_{i}=m j_{i}$. In the lowest Landau level limit, where we take the electron's mass to be zero, the momentum density conservation equation becomes the force balance equation from which one can derive $j_{i}$ in terms of $T_{i j}$. Combining with the charge conservation, one obtain the lowest Landau level Ward's identity [48]

$$
\partial_{t} \rho=\frac{4 i}{B}\left(\partial_{\bar{z}} \partial_{\bar{z}} T_{z z}-\partial_{z} \partial_{z} T_{\bar{z} \bar{z}}\right) .
$$

Using the Ward identity (A3), one can derive the first spectral sum rule for the lowest Landau level [41,48,53],

$$
\int_{0}^{\infty} \frac{d \omega}{\omega^{2}}\left[\rho_{T}(\omega)+\bar{\rho}_{T}(\omega)\right]=s_{4},
$$

where $s_{4}$ is the coefficient of $q^{4} \ell_{B}^{4}$ of the projected SSF, and the spectral functions $\rho_{T}$ and $\bar{\rho}_{T}$ are defined as [41]

$$
\begin{aligned}
& \rho_{T}(\omega)=\frac{1}{N} \sum_{n}\left|\left\langle n\left|\int d \mathbf{x} T_{z z}\right| 0\right\rangle\right|^{2} \delta\left(\omega-E_{n}\right), \\
& \bar{\rho}_{T}(\omega)=\frac{1}{N} \sum_{n}\left|\left\langle n\left|\int d \mathbf{x} T_{\bar{z} \bar{z}}\right| 0\right\rangle\right|^{2} \delta\left(\omega-E_{n}\right),
\end{aligned}
$$

where $N$ is the total number of electrons, $|0\rangle$ is the ground state, the sum is taken over all excited states $|n\rangle$ in the lowest Landau level, and $E_{n}$ is the energy of the state $|n\rangle$. The relation of Hall viscosity in terms of the retarded Green's functions of the stress tensor at zero spatial momentum is

$$
\omega \eta_{H}(\omega)=\left\langle T_{z z} T_{\bar{z} \bar{z}}\right\rangle_{\omega}-\left\langle T_{\bar{z} \bar{z}} T_{z z}\right\rangle_{\omega},
$$

with the definition of the retarded Green's function at frequency $\omega$,

$$
\langle A B\rangle_{\omega}=-i \int d t d^{2} \mathbf{x} e^{i \omega t} \Theta(t)\langle A(t, \mathbf{x}) B(0, \mathbf{0})\rangle .
$$

Using Eq. (A7), one can derive the second lowest Landau level sum rule,

$$
\int_{0}^{\infty} \frac{d \omega}{\omega^{2}}\left[\rho_{T}(\omega)-\bar{\rho}_{T}(\omega)\right]=\frac{\eta_{H}(0)-\eta_{H}(\infty)}{2 \bar{\rho}_{e}}=\frac{\mathcal{S}-1}{8},
$$


where we used the value of Hall viscosity at zero and high frequency,

$$
\eta_{H}(0)=\bar{\rho}_{e} \frac{\mathcal{S}}{4}, \quad \eta_{H}(\infty)=\frac{\bar{\rho}_{e}}{4} .
$$

By definition, both $\rho_{T}(\omega)$ and $\bar{\rho}_{T}(\omega)$ are non-negative, therefore the combination of sum rules (A4) and (A9) leads us to an inequality,

$$
s_{4} \geqslant \frac{|\mathcal{S}-1|}{8}
$$

which is the Haldane bound.

\section{APPENDIX B: GMP ALGEBRA OF THE DIRAC COMPOSITE FERMION SECTOR}

In this Appendix, we provide a detailed derivation of the GMP algebra of the composite fermion sector [Eq. (51) in the main text]. We ignore the trivial contribution of $B$ to the charge density operator of the CF section, and rewrite

$$
\rho^{(1)}=-\epsilon_{i j} \partial_{i} \tilde{\Pi}_{j},
$$

with

$$
\tilde{\Pi}_{i}=\frac{1}{B} \Pi_{i}+\frac{1}{4 \pi n} a_{i}=\frac{1}{B}\left[\tilde{\pi}_{i}-\left(\rho_{\mathrm{CF}}-\frac{B}{4 \pi n}\right) a_{i}\right],
$$

where we have used $\tilde{\pi}_{i}=-\frac{i}{2} \psi^{\dagger} \overleftrightarrow{\partial}_{i} \psi$. Using Eqs. (49) and (50), one obtains the following commutation relations by direct calculations:

$$
\begin{gathered}
{\left[\tilde{\pi}_{i}(\mathbf{x}), \tilde{\pi}_{j}(\mathbf{y})\right]=-i\left[\tilde{\pi}_{j}(\mathbf{x}) \frac{\partial}{\partial x^{i}}-\tilde{\pi}_{i}(\mathbf{y}) \frac{\partial}{\partial y^{j}}\right] \delta(\mathbf{x}-\mathbf{y}), \quad(\mathrm{B} 3)} \\
{\left[\tilde{\pi}_{i}(\mathbf{x}),-\left(\rho_{\mathrm{CF}}(\mathbf{y})-\frac{B}{4 \pi n}\right) a_{j}(\mathbf{y})\right]} \\
=i \rho_{\mathrm{CF}}(\mathbf{x}) a_{j}(\mathbf{x}) \frac{\partial}{\partial x^{i}} \delta(\mathbf{x}-\mathbf{y})+i \rho_{\mathrm{CF}}(\mathbf{x}) \partial_{i} a_{j}(\mathbf{x}) \delta(\mathbf{x}-\mathbf{y}), \\
{\left[-\left(\rho_{\mathrm{CF}}(\mathbf{x})-\frac{B}{4 \pi n}\right) a_{i}(\mathbf{x}), \tilde{\pi}_{j}(\mathbf{y})\right]} \\
=-i \rho_{\mathrm{CF}}(\mathbf{y}) a_{i}(\mathbf{y}) \frac{\partial}{\partial y^{j}} \delta(\mathbf{x}-\mathbf{y})-i \rho_{\mathrm{CF}}(\mathbf{y}) \partial_{j} a_{i}(\mathbf{y}) \delta(\mathbf{x}-\mathbf{y}), \\
{\left[-\left(\rho_{\mathrm{CF}}(\mathbf{x})-\frac{B}{4 \pi n}\right) a_{i}(\mathbf{x}),-\left(\rho_{\mathrm{CF}}(\mathbf{y})-\frac{B}{4 \pi n}\right) a_{j}(\mathbf{y})\right]} \\
=-i \frac{4 \pi n}{n-1} \epsilon^{i j} \delta(\mathbf{x}-\mathbf{y})\left(\rho_{\mathrm{CF}}(\mathbf{x})-\frac{B}{4 \pi n}\right)^{2} \\
=-i b(\mathbf{x}) \epsilon^{i j} \delta(\mathbf{x}-\mathbf{y})\left(\rho_{\mathrm{CF}}(\mathbf{x})-\frac{B}{4 \pi n}\right),
\end{gathered}
$$

where we have used the constraint

$$
\rho_{\mathrm{CF}}(\mathbf{x})=\frac{n-1}{4 \pi n} b(\mathbf{x})+\frac{B}{4 \pi n}
$$

to arrive at the last form in Eq. (B6). Combining Eqs. (B3), (B4), (B5), (B6), and the identity

$$
f(\mathbf{x}) \frac{\partial}{\partial x^{i}} \delta(\mathbf{x}-\mathbf{y})=f(\mathbf{y}) \frac{\partial}{\partial x^{i}} \delta(\mathbf{x}-\mathbf{y})-\partial_{i} f(\mathbf{x}) \delta(\mathbf{x}-\mathbf{y}),
$$

we obtain the commutation relation

$$
\begin{aligned}
{\left[\tilde{\Pi}_{i}(\mathbf{x}), \tilde{\Pi}_{j}(\mathbf{y})\right]=} & -\frac{i}{B}\left[\Pi_{j}(\mathbf{x}) \frac{\partial}{\partial x^{i}}-\Pi_{i}(\mathbf{y}) \frac{\partial}{\partial y^{j}}\right] \delta(\mathbf{x}-\mathbf{y}) \\
& +i \frac{B}{4 \pi n}\left[a_{j}(\mathbf{y}) \frac{\partial}{\partial x^{i}}-a_{i}(\mathbf{x}) \frac{\partial}{\partial y^{j}}\right] \delta(\mathbf{x}-\mathbf{y}) .
\end{aligned}
$$

Combining (B9) and (B1), we obtain the commutation relation of charge density in the momentum space [54],

$$
\left[\rho^{(1)}(\mathbf{k}), \rho^{(1)}(\mathbf{q})\right]=i(\mathbf{k} \times \mathbf{q}) \ell_{B}^{2} \rho^{(1)}(\mathbf{k}+\mathbf{q}),
$$

which is Eq. (51) of the main text.

\section{APPENDIX C: DENSITY OPERATOR IN THE SEMICLASSICAL FORMALISM}

In this Appendix, we provide a detailed derivation of the charge density operator of the CF sector in terms of $u_{m}$ operators. From Eqs. (B7) and (59) we have

$$
\dot{u}_{0}=\frac{n-1}{2 n k_{F}} \dot{b} .
$$

We rewrite the equation of motion for $u_{ \pm 1}$ as

$$
\begin{aligned}
\dot{u}_{1}= & -i\left(1+F_{1}\right) \frac{\bar{b} v_{F}}{k_{F}} u 1-v_{F}\left(1+F_{0}\right) \partial_{\bar{z}} u_{0} \\
& -v_{F}\left(1+F_{2}\right) \partial_{z} u_{2}+e_{\bar{z}}, \\
\dot{u}_{-1}= & i\left(1+F_{1}\right) \frac{\bar{b} v_{F}}{k_{F}} u_{-1}-v_{F}\left(1+F_{0}\right) \partial_{z} u_{0} \\
& -v_{F}\left(1+F_{2}\right) \partial_{\bar{z}} u_{2}+e_{\bar{z}} .
\end{aligned}
$$

We also need the equation of motion for $u_{ \pm 2}$ up to leading order in spatial derivatives

$$
\begin{aligned}
& \dot{u}_{2}=-i\left(1+F_{2}\right) \frac{\bar{b} v_{F}}{k_{F}} u_{2}, \\
& \dot{u}_{-2}=i\left(1+F_{2}\right) \frac{\bar{b} v_{F}}{k_{F}} u_{2} .
\end{aligned}
$$

Combing the time derivative of Eq. (65) and Eqs. (C2) and (C3), we obtain

$$
\begin{aligned}
\dot{\rho}^{(1)}= & -\frac{\dot{b}}{4 \pi n}+\frac{k_{F} v_{F} \bar{b}}{2 \pi B}\left(1+F_{1}\right)\left(\partial_{z} u_{1}+\partial_{\bar{z}} u_{-1}\right) \\
& -\frac{i v_{F} k_{F}^{2}}{2 \pi B}\left(1+F_{2}\right)\left(\partial_{z}^{2} u_{2}-\partial_{\bar{z}} u_{-2}\right) \\
& +\frac{i k_{F}^{2}}{2 \pi B}\left(\partial_{z} e_{\bar{z}}-\partial_{\bar{z}} e_{z}\right) .
\end{aligned}
$$

With the help of the Bianchi identity $\partial_{z} e_{\bar{z}}-\partial_{\bar{z}} e_{z}=-\frac{i}{2} \dot{b}$, the equation of motion for $u_{ \pm 2}$, and a combination of Eqs. (63) and $(\mathrm{C} 1)$, we obtain

$$
\dot{\rho}^{(1)}=\frac{\dot{b}}{4 \pi}\left(\frac{k_{F}^{2}}{B}-\frac{1}{n}-\frac{\bar{b}}{B} \frac{n-1}{n}\right)+\frac{k_{F}^{3}}{4 \pi B \bar{b}}\left(\partial_{z}^{2} \dot{u}_{2}+\partial_{\bar{z}}^{2} \dot{u}_{-2}\right),
$$

which is Eq. (66) in the main text. 
[1] D. C. Tsui, H. L. Stormer, and A. C. Gossard, Two-Dimensional Magnetotransport in the Extreme Quantum Limit, Phys. Rev. Lett. 48, 1559 (1982).

[2] R. B. Laughlin, Anomalous Quantum Hall Effect: An Incompressible Quantum Fluid with Fractionally Charged Excitations, Phys. Rev. Lett. 50, 1395 (1983).

[3] J. K. Jain, Composite-Fermion Approach for the Fractional Quantum Hall Effect, Phys. Rev. Lett. 63, 199 (1989).

[4] F. Wilczek, Magnetic Flux, Angular Momentum, and Statistics, Phys. Rev. Lett. 48, 1144 (1982).

[5] B. Halperin, Theory of the quantized Hall conductance, Helv. Phys. Acta 56, 75 (1983).

[6] S. M. Girvin and A. H. MacDonald, Off-Diagonal Long-Range Order, Oblique Confinement, and the Fractional Quantum Hall Effect, Phys. Rev. Lett. 58, 1252 (1987).

[7] B. I. Halperin, P. A. Lee, and N. Read, Theory of the half-filled Landau level, Phys. Rev. B 47, 7312 (1993).

[8] W. Kang, H. L. Stormer, L. N. Pfeiffer, K. W. Baldwin, and K. W. West, How Real are Composite Fermions? Phys. Rev. Lett. 71, 3850 (1993).

[9] S. A. Kivelson, D.-H. Lee, Y. Krotov, and J. Gan, Compositefermion Hall conductance at $v=\frac{1}{2}$, Phys. Rev. B 55, 15552 (1997).

[10] D. Kamburov, Y. Liu, M. A. Mueed, M. Shayegan, L. N. Pfeiffer, K. W. West, and K. W. Baldwin, What Determines the Fermi Wave Vector of Composite Fermions? Phys. Rev. Lett. 113, 196801 (2014).

[11] D. T. Son, Is the Composite Fermion a Dirac Particle? Phys. Rev. X 5, 031027 (2015).

[12] D. X. Nguyen, S. Golkar, M. M. Roberts, and D. T. Son, Particle-hole symmetry and composite fermions in fractional quantum Hall states, Phys. Rev. B 97, 195314 (2018).

[13] D. Shahar, D. C. Tsui, M. Shayegan, E. Shimshoni, and S. L. Sondhi, Evidence for charge-flux duality near the quantum Hall liquid-to-insulator transition, Science 274, 589 (1996).

[14] J. Wang, Dirac Fermion Hierarchy of Composite Fermi Liquids, Phys. Rev. Lett. 122, 257203 (2019).

[15] We will leave an explicit derivation of the Haldane bound to Appendix A.

[16] H. Goldman and E. Fradkin, Dirac composite fermions and emergent reflection symmetry about even-denominator filling fractions, Phys. Rev. B 98, 165137 (2018).

[17] F. D. M. Haldane, "Hall viscosity" and intrinsic metric of incompressible fractional Hall fluids, arXiv:0906.1854.

[18] A. Gromov and D. T. Son, Bimetric Theory of Fractional Quantum Hall States, Phys. Rev. X 7, 041032 (2017).

[19] G. Y. Cho, Y. You, and E. Fradkin, Geometry of fractional quantum Hall fluids, Phys. Rev. B 90, 115139 (2014).

[20] A. Gromov, G. Y. Cho, Y. You, A. G. Abanov, and E. Fradkin, Framing Anomaly in the Effective Theory of the Fractional Quantum Hall Effect, Phys. Rev. Lett. 114, 016805 (2015).

[21] C. Hoyos and D. T. Son, Hall Viscosity and Electromagnetic Response, Phys. Rev. Lett. 108, 066805 (2012).

[22] D. X. Nguyen and D. T. Son, Algebraic approach to fractional quantum Hall effect, Phys. Rev. B 98, 241110(R) (2018).

[23] F. D. M. Haldane, Geometrical Description of the Fractional Quantum Hall Effect, Phys. Rev. Lett. 107, 116801 (2011).
[24] The Landé factor is introduced for convenience and does not change physical quantities computed in this paper, for example the static structure factor.

[25] D. T. Son, Newton-Cartan geometry and the quantum Hall effect, arXiv:1306.0638

[26] M. Geracie, D. T. Son, C. Wu, and S.-F. Wu, Spacetime symmetries of the quantum Hall effect, Phys. Rev. D 91, 045030 (2015).

[27] K. Prabhu and M. M. Roberts, Electrons and composite Dirac fermions in the lowest Landau level, arXiv:1709.02814.

[28] We take $\mathfrak{g}=2$ only for convenience in which the mathematical treatment of the lowest Landau level limit is simplified. A different value of $\mathfrak{g}$ only modifies the chemical potential by a constant value [26] that does not change the physical conclusions in this paper.

[29] D. X. Nguyen, A. Gromov, and D. T. Son, Fractional quantum Hall systems near nematicity: Bimetric theory, composite fermions, and Dirac brackets, Phys. Rev. B 97, 195103 (2018).

[30] Which was also found in the IQH experiment of graphene after dividing the experimental result of $\sigma_{x y}$ by the total number of spins and valleys [55].

[31] S. Golkar, M. M. Roberts, and D. T. Son, Effective field theory of relativistic quantum Hall systems, J. High Energy Phys. 2014, 138 (2014).

[32] The similar effective action of nonrelativistic IQH states can be found in [56].

[33] Note that, due to the additional dipole term, one need to shift $a_{0} \rightarrow a_{0}+\frac{1}{2} \frac{k_{F}}{v_{F}} v_{i} v^{i}$ and $a_{i} \rightarrow a_{i}-\frac{k_{F}}{v_{F}} v_{i}$ as explained in [12]. However, the extra terms are higher derivative and do not modify the results of this paper.

[34] Due to the definition of $\omega_{0}$, there is an extra contribution to the charge density with the form $\frac{1}{\sqrt{g}} \partial_{i} \partial^{i}(\cdots)$. However, this term will not contribute to the Wen-Zee shift on a closed manifold.

[35] Note the definition of $\tilde{A}$ in Eq. (2).

[36] X. G. Wen and A. Zee, Shift and Spin Vector: New Topological Quantum Numbers for the Hall Fluids, Phys. Rev. Lett. 69, 953 (1992).

[37] S. M. Girvin, A. H. MacDonald, and P. M. Platzman, Magnetoroton theory of collective excitations in the fractional quantum Hall effect, Phys. Rev. B 33, 2481 (1986).

[38] J. Avan and A. Jevicki, Classical integrability and higher symmetries of collective string field theory, Phys. Lett. B 266, 35 (1991).

[39] A. Gerasimov, A. Marshakov, A. Mironov, A. Morozov, and A. Orlov, Matrix models of two-dimensional gravity and Toda theory, Nucl. Phys. B 357, 565 (1991).

[40] S. Iso, D. Karabali, and B. Sakita, Fermions in the lowest Landau level. Bosonization, $W_{\infty}$ algebra, droplets, chiral bosons, Phys. Lett. B 296, 143 (1992).

[41] S. Golkar, D. X. Nguyen, M. M. Roberts, and D. T. Son, Higher-Spin Theory of the Magnetorotons, Phys. Rev. Lett. 117, 216403 (2016).

[42] It has been shown that the the semiclassical approach gives the same results of electromagnetic response as the random phase approximation calculation [45].

[43] J. M. Luttinger, Fermi surface and some simple equilibrium properties of a system of interacting fermions, Phys. Rev. 119 , 1153 (1960).

[44] F. D. M. Haldane, Luttinger's theorem and bosonization of the fermi surface, in Perspectives in Many-Particle Physics, edited 
by R. A. Broglia, J. R. Schrieffer, and P. F. Bortignon (NorthHolland, Amsterdam, 1994), pp. 5-30.

[45] D. X. Nguyen and A. Gromov, Exact electromagnetic response of Landau level electrons, Phys. Rev. B 95, 085151 (2017).

[46] One can derive the same equation of motion using the Boltzmann kinetic equation $[12,45]$.

[47] The mixed correlation $\left\langle\rho^{(1)}(-\mathbf{q}) \rho^{(2)}(\mathbf{q})\right\rangle$ vanishes.

[48] D. X. Nguyen, D. T. Son, and C. Wu, Lowest Landau level stress tensor and structure factor of trial quantum Hall wave functions, arXiv:1411.3316.

[49] D. X. Nguyen, T. Can, and A. Gromov, Particle-Hole Duality in the Lowest Landau Level, Phys. Rev. Lett. 118, 206602 (2017).

[50] S.-F. Liou, F. D. M. Haldane, K. Yang, and E. H. Rezayi, Chiral Gravitons in Fractional Quantum Hall Liquids, Phys. Rev. Lett. 123, 146801 (2019).
[51] F. D. M. Haldane, E. H. Rezayi, and K. Yang, Graviton chirality and topological order in the half-filled Landau level, arXiv:2103.11019.

[52] D. X. Nguyen and D. T. Son, Probing the spin structure of the fractional quantum Hall magnetoroton with polarized Raman scattering, Phys. Rev. Res. 3, 023040 (2021).

[53] The extra terms [second line in Eq. (B9)] disappear when we compute the curls in $\left[\rho^{(1)}(\mathbf{x}), \rho^{(1)}(\mathbf{y})\right]$.

[54] The upper limit $\infty$ here means a frequency that is much higher than the Coulomb gap but also smaller the the cyclotron gap.

[55] V. P. Gusynin and S. G. Sharapov, Unconventional Integer Quantum Hall Effect in Graphene, Phys. Rev. Lett. 95, 146801 (2005).

[56] A. G. Abanov and A. Gromov, Electromagnetic and gravitational responses of two-dimensional noninteracting electrons in a background magnetic field, Phys. Rev. B 90, 014435 (2014). 